\title{
REVISIONES
}

\section{Conducta adaptativa y discapacidad intelectual: 50 años de historia y su incipiente desarrollo en la educación en Chile}

\author{
Adaptive behavior and intellectual disability: \\ 50 years of history and early development in education in Chile \\ Conduta adaptativa e deficiência intelectual: 50 anos de história \\ e seu incipiente desenvolvimento na educação no Chile \\ Delfin Montero $^{1}$, Juan Lagos ${ }^{2}$ \\ ${ }^{1}$ Universidad de Deusto, Facultad de Psicología y Educación, \\ Avenida de las Universidades, 24, Bilbao, España, meducaes@fice.deusto.es \\ ${ }^{2}$ Universidad Católica del Maule, Facultad de Ciencias de la Educación, \\ Avenida San Miguel, 3605, Talca, Chile, jlagos@ucm.cl
}

\begin{abstract}
RESUMEN
El artículo pretende describir la génesis del constructo conducta adaptativa, su vinculación con la noción de discapacidad intelectual y poner de relieve la importancia de la conducta adaptativa como criterio para el diagnóstico de discapacidad intelectual. Asimismo, se caracterizará el constructo conducta adaptativa tanto en lo que concierne a sus aspectos legales como a su uso para el diagnóstico en las orientaciones técnicas y los formularios únicos propuestos por el Ministerio de Educación de Chile. Finalmente, se plantea una discusión sobre los aciertos y desaciertos del incipiente desarrollo del uso del constructo conducta adaptativa, en lo que concierne a su utilización en personas con discapacidad intelectual específicamente en contextos educativos.
\end{abstract}

Palabras clave: conducta adaptativa, discapacidad intelectual, diagnóstico de discapacidad intelectual.

\begin{abstract}
The main aim of this article is to describe the genesis of the construct adaptive behavior, its relation with the idea of intellectual disability and to remark the importance of the adaptive behavior as criteria for the diagnosis of intellectual disability. Also will be characterized the adaptive behavior construct in what concerns to legal aspects, as well as its use for the diagnosis in the technical orientations and the unique forms proposed by the Ministry of Education in Chile. Finally, we present a discussion about the strengths and shortcomings of the incipient development of the use of adaptive behavior construct, with respect to their use in people with intellectual disabilities specifically in educational settings.
\end{abstract}

Key words: adaptive behavior, intellectual disability, intellectual disability diagnosis.

\section{RESUMO}

Descreve-se a gênese do instrumento, Conduta Adaptativa, sua vinculação com a noção de deficiência intelectual e dá relevância à importância de conduta adaptativa como critério para o diagnóstico de deficiência intelectual. Assim considerando, caracteriza-se o referido instrumento em relação aos seus aspectos legais e uso para o diagnóstico nas orientações técnicas e nos formulários exclusivos, propostos pelo Ministério de Educação do Chile. Finalmente, propõe-se uma discussão sobre acertos e erros do incipiente desenvolvimento do uso do instrumento, Conduta Adaptativa, no que tange à sua utilização com pessoas com deficiência intelectual, em contextos educativos específicos.

Palavras chave: conduta adaptativa, deficiência intelectual, diagnóstico de deficiência intelectual. 


\section{LA CONDUCTA ADAPTATIVA EN EL CAMPO DE LA DISCAPACIDAD INTELECTUAL}

Desde que Heber (1959) introdujera el constructo de conducta adaptativa por primera vez en la definición de deficiencia mental (de ahora en adelante discapacidad intelectual) propuesta por la Asociación Americana sobre Deficiencia Mental (AAMD), no ha dejado de producir entre los investigadores y académicos un gran número de controversias, ya sea por su dificultad para definirlo (Clausen, 1972), por las limitaciones para evaluarlo, debido a la imposibilidad de establecer patrones de adaptación absolutos (Baumeister \& Muma, 1975), y por el poco empleo de este constructo en la práctica profesional (Zigler, Balla \& Hodapp, 1984). Estas observaciones críticas al constructo de conducta adaptativa y su consideración como parte de la discapacidad intelectual, no obstante, no ha sido impedimento para continuar la investigación acerca de su ámbito conceptual, su aplicación para el diagnóstico y evaluación, así como para la determinación de dispositivos de apoyo a las personas con discapacidad intelectual. Por el contrario, han alentado el incremento del trabajo entorno de ella y han posibilitado mejoras en su aplicación.

En opinión de algunos investigadores, el constructo de conducta adaptativa ha contribuido a mejorar significativamente el diagnóstico, clasificación y planificación de apoyos (Bruininks, Thurlow \& Gilman, 1987; Luckasson et al., 1992; Luckasson et al., 2003; Jacobson, 2006; Schalock et al., 2010). Este trabajo ha permitido incrementar la congruencia entre la conducta adaptativa, tal y como la formula la Asociación Americana de Discapacidad Intelectual y del Desarrollo (AAIDD, antes AAMD) (Schalock et al., 2010), con los modelos conceptuales que se describen en los dominios de competencia personal en los trabajos de Greenspan (Greenspan, 1981; Greenspan \& Granfield, 1992; Greenspan, Switzky \& Granfield, 1996; Greenspan \& Driscoll, 1997); ha posibilitado la relación con algunas teorías de inteligencia (Woodcock \& Muñoz-Sandoval, 1993; Schalock, 1999) y con la capacidad de autodeterminación (Wehmeyer, Abery, Mithaug, \& Stancliffe, 2003), y, finalmente, también ha favorecido una relación más estrecha con los principios de calidad de vida de personas con discapacidad intelectual (Schalock, 2001; Schalock, 2003).

Asimismo, la noción de conducta adaptativa no sólo encuentra eco en la Asociación Americana de Discapacidad Intelectual y del Desarrollo (Schalock et al., 2010), sino que es utilizada en los criterios de diagnóstico en las últimas ediciones del Manual Diagnóstico y Estadístico de los Trastornos Mentales (DSM IV-TR), compilado por la Asociación Americana de Psiquiatría (APA) (1994, 2000). Tanto AAIDD como la APA son dos instituciones de referencia obligada en la investigación y en la praxis profesional en el campo de la discapacidad intelectual.

Con la finalidad de ofrecer una breve reseña histórica del constructo de conducta adaptativa como uno de los tres criterios para la evaluación de la discapacidad intelectual, este artículo pretende visualizar la trayectoria histórica de la conducta adaptativa y comprender su desarrollo paralelamente al concepto de discapacidad intelectual. Para desarrollar estos propósitos, el artículo contiene en primer lugar una reconstrucción histórica del constructo de conducta adaptativa; en segundo lugar, describe el uso en la política e instrumentación educativa; para, finalmente, puntualizar observaciones críticas sobre el uso del constructo en cuestión en el sistema educativo chileno. 


\section{RESEÑA HISTÓRICA DE LA CONDUCTA ADAPTATIVA EN EL CAMPO DE LA DISCAPACIDAD INTELECTUAL}

La discapacidad intelectual antes de 1900 se diagnosticaba en términos de un conjunto de rasgos que caracterizaban la adaptación de los individuos al entorno social, usando expresiones tales como "competencia social", "habilidades de entrenamiento", "normas sociales", "capacidad de arreglárselas por uno mismo en la vida" y "adaptabilidad al entorno" (Nihira, 1999). La conducta adaptativa, al ser incluida en la definición de retraso mental presentada por la AAMD en los años de 1959, 1961 y posteriormente en cada una de las ediciones hasta la actual del año 2010, ha permitido neutralizar el efecto reductivo de la medición del funcionamiento intelectual, tal como lo miden los tests de inteligencia, como referente exclusivo para el diagnóstico de discapacidad intelectual, en un intento por modular el predominio que estas escalas tuvieron durante la primera mitad del siglo XX.

Como ha sido señalado, la evolución ent orno al constructo de conducta adaptativa, comenzó a configurarse en la segunda mitad del siglo XX. Así, en la década de los sesenta, específicamente en el año 1964, la AAMD, en conjunto con el Parsons State Hospital and Training Center y con el apoyo de una beca concedida por el Nacional Institute of Mental Health, desarrolló una investigación para demostrar la función de la conducta adaptativa en problemas psiquiátricos, como medio para establecer un nuevo sistema de clasificación dentro de la discapacidad intelectual. El producto de dicho trabajo fue la Adaptive Behavior Checklist para niños y adultos (Nihira, Foster, Shellhaas \& Leland, 1968) y desde entonces la conducta adaptativa se orientó a fomentar una perspectiva educativa sobre los individuos, más que solo como clasificación y etiquetado de carácter puramente clínico.

Durante los años sesenta, asimismo, se produjo una aproximación al constructo de conducta adaptativa, debido a que ésta fue una década de preocupación y conciencia social respecto a la educabilidad de personas con discapacidad intelectual. Quizás en relación con ello se pueda entender que el Presidente de los EE.UU., John Fitzgerald Kennedy, fuera nombrado primer Presidente del Comité de Retraso Mental, a lo que se suma el que una hermana suya presentaba discapacidad intelectual, y que en febrero de 1963 Kennedy recomendara al Congreso de los Estados Unidos "Un Programa Nacional para Combatir el Retraso Mental". La suma de estos hechos, entre otros, dio como resultado el que nunca antes hubo tantos recursos centrados en la atención al problema de la discapacidad intelectual como en esta década.

Por otra parte, la inclusión de la conducta adaptativa en la definición de la AAMD incrementó la urgencia de generar instrumentos de evaluación para el diagnóstico y planificación de apoyos en la práctica educativa. Esto permitió establecer objetivos, programas de formación y de evaluación; de igual forma, la conducta adaptativa promovió la desinstitucionalización y rehabilitación de personas con discapacidad intelectual en la comunidad (Nihira, 1999). En una revisión realizada por Horn \& Fuchs (1987), se menciona que a lo largo de la historia de las sociedades, cuando éstas demostraron disposición para el desarrollo de programas para personas con discapacidad intelectual, dichos programas se basaban en el concepto de conducta adaptativa para definir el contenido de los mismos.

Una década más tarde, vale decir, durante los años setenta, el constructo de conducta adaptativa consideraba los principios de normalización (Wolfensberger, 1972) para personas 
con discapacidad intelectual, quienes debían poseer las habilidades básicas requeridas para su función en un medio reducido. Progresivamente, las investigaciones estuvieron dedicadas a la identificación de habilidades necesarias para una plena integración social (Meyers, Nihira, \& Zetlin, 1979; Horn \& Fuchs, 1987).

En 1975 la educación de personas con discapacidad intelectual exigía como deber del Estado aplicar pruebas no discriminatorias en la evaluación y derivación educativa; de hecho, la conducta adaptativa se incorpora en la ley de EE.UU. PL-94-142 sobre personas con discapacidad. La cuestión central de la evaluación no discriminatoria evitó la desproporcionada identificación y la derivación educativa de niños de minorías étnicas a grupos de educación especial (Nihira, 1999). En este contexto, la evaluación de la conducta adaptativa facilitó la decisión acerca de dónde se educarían las personas que presentaban discapacidad intelectual en coherencia con sus posibilidades cognitivas y conductuales. Además, permitió la identificación del desarrollo social de los niños fuera del entorno académico.

Como puede constatarse, y según como lo sostiene Nihira (1999), la conducta adaptativa estuvo involucrada en la controversia legal y educacional, jugando un rol más importante que lo atribuido a los test de cociente intelectual, por lo que se usó para la ubicación diferencial de los niños en las escuelas públicas. Incluso en algunos estados se mandató incluir la valoración de la conducta adaptativa como una evaluación multidimensional para equilibrar finalmente los potenciales de niños, niñas y jóvenes.

De este modo, el uso del constructo de conducta adaptativa vino a ser una potente herramienta de diagnóstico en la evaluación no discriminatoria, al punto que durante los años setenta se determinó utilizarlo en la identificación y clasificación de niños escolares para efectos de la entrega de recursos proveniente de la administración del Estado (Mercer, 1973). Estas razones son de histórica importancia, pues tuvieron un profundo efecto posterior en la investigación sobre la conducta adaptativa y en qué aspectos debían gravitar centralmente las escalas para su evaluación.

Seguidamente, en la década de los ochenta, se desarrolló una cantidad sustancial de investigaciones que utilizaron las mediciones de conducta adaptativa para la descripción de qué habilidades deben presentarse en distintos escenarios de la vida cotidiana (Hawkins \& Cooper, 1990). Además, en los ochenta las medidas de evaluación de la conducta adaptativa continuaron siendo efectivas para desarrollar programas de instrucción y preparar a personas para vivir en espacios pequeños y elaborar programas transitorios para la vida adulta (Silverman, Silver, Sersen, Lubin \& Schwart, 1986).

Algunos investigadores encontraron en la conducta adaptativa información demasiado imprecisa, de cuestionable validación para efecto de clasificación (Zigler, Balla \& Hodapp, 1984) y de limitado uso en la escuela (Witt \& Martens, 1984). En respuesta a estos alcances, en la década de los ochenta la mirada investigativa se dirigió hacia la compresión, la naturaleza, la estructura y el desarrollo de la conducta adaptativa (Nihira, 1999).

Otro acontecimiento destacado de los años ochenta fue la definición aportada por el Manual de la AAMD (Grossman, 1983). En el transcurso de las décadas anteriores y posteriores a esta definición existieron otras, pero por ser ésta una de las más relevantes, nos permitimos referirla in extenso:

La conducta adaptativa hace referencia a la calidad de las ejecuciones diarias que dan respuesta a las demandas ambientales. La calidad de la adaptación general está medida por el nivel de inteligencia; por lo tanto, el significado de los dos conceptos se superpone en significado. Es 
evidente, sin embargo, considerando a la definición de la conducta adaptativa con su énfasis sobre las respuestas cotidianas, que la conducta adaptativa se refiere a lo que las personas hacen para cuidar de sí mismas y para relacionarse con otros en la vida diaria, más bien que al potencial abstracto implícito en el concepto de inteligencia (Grossman, 1983: 42).

Por otra parte, algunas de las tendencias en las investigaciones en los años ochenta aportaron información relevante para comprender la naturaleza de la conducta adaptativa. Algunos estudios longitudinales y transversales indicaban claramente diferencias en el desarrollo mental para el pronóstico en la conducta adaptativa de los distintos grados de discapacidad intelectual (Grossman, 1983). Además, ciertos dominios de la conducta adaptativa estaban caracterizados en la trayectoria del desarrollo mental. Por ejemplo, personas con moderada discapacidad intelectual demostraban un rápido desarrollo en las habilidades personales durante su temprana infancia, mientras que las habilidades de comunicación y habilidades para la vida en comunidad continúan su desarrollo durante la adolescencia y durante la vida adulta (Janicki \& Jacobson, 1986; Eyman \& Widaman, 1987). Estos estudios ayudaron a identificar los requisitos específicos para proporcionar los servicios y apoyos relacionando diferentes edades y grados de las personas con discapacidad intelectual.

Finalizando el milenio, en los años noventa, la Asociación Americana de Retraso Mental (AAMR, ex AAMD) (Luckasson et al., 1992) presenta su nuevo Manual, en el cual sustituye el constructo de conducta adaptativa por el de "habilidades adaptativas", el que de acuerdo con Greenspan \& Driscoll (1997) tiene el mismo significado. Pese a la opinión de estos autores, las habilidades adaptativas consiguen relativizar el constructo de conducta adaptativa, constituyendo un cambio debido en parte a las dificultades presentadas por la conceptualización de la conducta adaptativa y a los problemas relacionados con la medición de un constructo tan extenso (Luckasson et al., 1992).

En la edición de 1992, las habilidades adaptativas son presentadas en diez áreas, las cuales vienen enunciadas en un sistema descriptivo desarrollado por Ford (Ford, Schnorr, Meyer, Davern, Black \& Dempsey, 1989). Las áreas aludidas son las siguientes: comunicación, cuidado personal, habilidades de vida en el hogar, habilidades sociales, utilización de la comunidad, autorregulación, salud y seguridad, habilidades académicas funcionales, ocio y trabajo.

Asimismo, en los años noventa se destacan investigaciones sobre marcos de referencia del constructo y su estructura, tal es el caso de los trabajos de Widaman \& McGrew (1996); Thompson, McGrew \& Bruininks (1999). Hacia fines de los años noventa y comienzos del 2000, los investigadores enfatizan la importancia de la evaluación de la conducta adaptativa como un criterio específico para el diagnóstico de la discapacidad intelectual.

A inicios del siglo XXI, específicamente en el año 2002, se publica la décima edición, AAMR (Luckasson et al., 2002) en la cual se considera la conducta adaptativa como una dimensión agregada al modelo anterior (Luckasson et al., 1992). Cabe subrayar que en este Manual se vuelve a utilizar el constructo de conducta adaptativa, eliminándose el de habilidades adaptativas, pero el constructo al que se alude en el Manual se expresa en términos de habilidades adaptativas conceptuales, prácticas y sociales, lo que es congruente con los trabajos sobre competencia personal, social e intelectual que Stephen Greenspan ha propuesto en sus investigaciones (Greenspan, 1981; Greenspan \& Granfield, 1992; Greenspan, Switzky \& Granfield, 1996; Greenspan \& Driscoll, 1997). 
A finales del siglo XX e inicios del XXI, los investigadores han destacado empírica y teóricamente que el constructo de conducta adaptativa se vincula con otros, tales como el de inteligencia (Woodcock, Muñoz-Sandoval 1993; Schalock, 1999; y Schalock, 2001) y el de autodeterminación (Stancliffe \& Abery, 2003). En relación con este último, Montero (2003) ha señalado que cualquiera que analice una herramienta lo suficientemente amplia de conducta adaptativa, se dará cuenta de que autodeterminarse en la propia vida difícilmente puede hacerse sin partir de la sólida base que proporciona el conjunto de destrezas que precisa un ciudadano para enfrentarse con un mínimo de éxito a las demandas adaptativas de cada día.

Por otra parte, es necesario hacer mención a la importancia que se le atribuye a la conducta adaptativa como predictor de la calidad de vida, al permitir que una persona con discapacidad intelectual adquiera las destrezas necesarias que promueven una conducta más independiente, lo que implica incluir en los programas de apoyo al menos uno de los principios de calidad de vida en personas con discapacidad intelectual. Dichos principios hacen mención a factores ambientales y personales tales como: las relaciones íntimas, la vida familiar, las amistades, el trabajo, el vecindario, la ciudad de residencia, la casa, la educación, la salud y el estándar de vida (Verdugo y Schalock, 2001).

Finalmente, en la décimo primera edición del Manual de la AAIDD (Schalock et al., 2010), se mantiene la conducta adaptativa como una de las cinco dimensiones del modelo de discapacidad intelectual, lo que refuerza la idea de la AAMD (Grossman, 1983), que sugería homologar el modelo de discapacidad intelectual al de desarrollo humano de la Organización Mundial de la Salud, expresado en la Clasificación Internacional del Funcionamiento, de la Discapacidad y de la Salud.

La aproximación histórica al concepto de conducta adaptativa espera abrir un debate sobre cómo lo entendemos, cómo se desarrolla y cómo se genera conocimiento acerca de los estándares mínimos necesarios para que una persona con discapacidad intelectual se integre socialmente, para que sea socialmente valorada y para que alcance niveles apropiados de autodeterminación.

\section{LA CONDUCTA ADAPTATIVA Y SU FIGURA EN LA POLÍTICA EDUCATIVA EN CHILE}

A continuación se examina cómo la conducta adaptativa está siendo incorporada en los aspectos de tipo legal, de orientación para la evaluación de personas con discapacidad intelectual y, finalmente, en los Formularios Únicos presentados por el Ministerio de Educación de Chile, específicamente de la Unidad de Educación Especial.

A finales del año 2009 se promulga el Decreto $\mathrm{N}^{\circ} 170$, con fecha de publicación del 14 de mayo de 2009, correspondiente al reglamento de la Ley $\mathrm{N}^{\circ} 20.201$, en el cual se fijan normas para clasificar a los alumnos con necesidades educativas especiales que serán beneficiarios de las subvenciones (recursos económicos diferenciales) para la Educación Especial. En el artículo $\mathrm{N}^{\mathrm{o}} 63$ se hace mención a las limitaciones de la conducta adaptativa para el diagnóstico de discapacidad intelectual, considerando que dichas limitaciones:

Afectan el funcionamiento esperado en la vida diaria, en la capacidad para responder a cambios vitales, a las demandas del ambiente y cuando los resultados en las escalas anteriormente 
señaladas se encuentran a más de dos desviaciones estándar por debajo de la media en la evaluación de uno de los tres componentes: conceptual, práctico y social o en una puntuación global de éstos (Decreto $N^{\circ}$ 2009: 17).

La declaración contenida en el artículo recién citado es especialmente importante, puesto que constituye la primera ocasión en que formalmente se hace una exigencia sobre la evaluación de la conducta adaptativa en personas con "discapacidad intelectual de tipo severa" y para el "diagnóstico de coeficiente intelectual en el rango límite" (Artículo $\mathrm{N}^{\circ} 47$ letra b). Aún más, el contenido del artículo $\mathrm{N}^{\circ} 63$ ofrece además una clara distinción relativa a la evaluación y promoción de habilidades prácticas, sociales y/o conceptuales.

La exigencia jurídica en orden a evaluar la conducta adaptativa e incorporar los resultados de dicha evaluación como uno de los requisitos de diagnóstico (Artículo No 57), representa un reconocimiento expreso de la legislación chilena, que se alinea con los criterios internacionalmente aceptados. Del mismo modo, el reconocimiento jurídico mencionado es coherente con el criterio de diagnóstico de la AAIDD (Luckasson et al., 2002; Schalock et al., 2010) y de la Asociación Americana de Psiquiatría, a través del DSM IV-TR (1994/2000).

A fines del mismo año 2009, la Unidad de Educación Especial del Ministerio de Educación de Chile, dispuso un documento denominado "Orientaciones Técnicas para la Evaluación Diagnóstica de Estudiantes que presentan Necesidades Educativas Especiales asociadas a Discapacidad Intelectual" (de ahora en adelante Orientaciones Técnicas) que describe un conjunto de aspectos relacionados con la conducta adaptativa, entre los cuales se cuentan sugerencias de instrumentos, especificación de edades sobre las cuales aplicar evaluaciones, la operativización de las limitaciones en la conducta adaptativa que debe presentar una persona para ser diagnosticada con discapacidad intelectual, entre otros, concordantemente con la definición ofrecida por la AAMR (Luckasson et al., 2002).

Con estas Orientaciones Técnicas se pretende entregar a quienes diagnostican discapacidad intelectual, fundamentalmente psicólogos, algunos elementos que les permitan un diagnóstico certero y conforme a criterios comunes. El documento de Orientaciones Técnicas ha sido formulado recogiendo el modelo multidimensional de discapacidad intelectual planteado por la AAIDD (en ese entonces AAMR) (Luckasson et al., 2002), basándose además en el enfoque conceptual de la OMS, presentado a través del modelo de funcionamiento humano (OMS, 2001).

En cuanto a la evaluación diagnóstica, las Orientaciones Técnicas precisan que este proceso al interior del ámbito educativo debe ser concebido con miras a fijar una enseñanza más efectiva y a proporcionar los apoyos que requiere un o una estudiante para progresar, aprender y participar. Para establecer el diagnóstico de discapacidad intelectual, las Orientaciones Técnicas indican que se deben cumplir tres requisitos, a saber:

a. limitaciones significativas del funcionamiento intelectual;

b. limitaciones significativas de la conducta adaptativa, y;

c. edad no superior a 18 años.

En lo referente al segundo criterio señalado, esto es, al que concierne a "limitaciones significativas en la conducta adaptativa", las Orientaciones Técnicas afirman que en Chile 
no existen escalas de conducta adaptativa estandarizadas, carencia que produce como consecuencia el que los antecedentes referidos a estas habilidades sean recabados por medio de instrumentos internacionales o a través del así llamado "juicio clínico" de los psicólogos. En este sentido, se agrega en el documento de Orientaciones Técnicas que mientras en Chile no existan escalas adaptadas y validadas con baremos nacionales para la población con y sin discapacidad, se recomienda el uso preferente de los instrumentos que a continuación se mencionan en la Tabla 1:

\section{Tabla 1}

Instrumentos de evaluación de la conducta adaptativa sugeridos en Chile

\begin{tabular}{|l|l|}
\hline Test & \multicolumn{1}{|c|}{ Rango edad } \\
\hline $\begin{array}{l}\text { Inventario para la planificación de servicios y la programación individual } \\
\text { (ICAP) (Montero, 1993). }\end{array}$ & Todas las edades \\
\hline Escala de Madurez Social de Vineland, adaptación chilena (Otero, 1959). & Todas las edades \\
\hline $\begin{array}{l}\text { Escala de Intensidad de Apoyos-SIS. Adaptación española (Verdugo, Arias, } \\
\text { \& Ibañez, 2007) (Supports Intensity Scale (SIS), AAIDD, 2004). }\end{array}$ & A partir de los 16 años \\
\hline
\end{tabular}

Elaboración propia.

En la medida en que la consideración del constructo conducta adaptativa efectuada en las Orientaciones Técnicas implica la referencia al uso de escalas psicométricas, puede estimarse que éste constituye un acierto adicional del documento en tanto permite una articulación más óptima entre la evaluación, el diagnóstico y la generación de apoyos para personas con discapacidad intelectual.

A fines del año 2010, el Ministerio de Educación de Chile presentó una propuesta para implementar un proceso de evaluación que incluye a un conjunto diverso de actores y se refiere a las necesidades educativas especiales transitorias y permanentes, entre otras. Esta propuesta comprende en parte los llamados "Formularios Únicos" cuya disposición para el ejercicio profesional resulta ser de gran ayuda en la medida en que exigen la integración formal de la familia al proceso, a través de una autorización explícita para su realización, y en cuanto regulan los instrumentos de evaluación requeridos para los correspondientes diagnósticos y la definición de los apoyos necesarios.

En los "Formularios Únicos" la presencia de la conducta adaptativa tiene lugar en las necesidades educativas especiales transitorias, específicamente en el "rango límite", y necesidades educativas especiales permanentes, particularmente en "discapacidad intelectual" y "discapacidades múltiples". En ambos casos, los Formularios sugieren el uso de las pruebas presentadas en la Tabla $\mathrm{N}^{\mathrm{o}} 1$ y dejan abierta la posibilidad de incorporar "otras" pruebas (formales o informales), sin más especificación.

En el caso del Formulario Único referido a Discapacidad Múltiple, se consideran los mismos instrumentos propuestos en la Tabla $\mathrm{N}^{\circ} 1$-presumiendo que el "CAP" al que alude el Formulario corresponde al ICAP-, y se agrega a ellos el "Choosing Outcomes and Accommodations for Children" (COACH) de Giangreco, Cloninger \& Iverson (1998). 
En la referencia a los autores del COACH se omite el nombre de Virginia Salce Iverson, quien participó también en su elaboración. Junto con esta omisión, quizá debida a un problema de digitación, llama la atención que el año de edición del COACH corresponda al 2008, puesto que según las evidencias de las que se dispone existen dos ediciones del instrumento, la primera en el año 1993 y la segunda en el año 1998. De hecho, la información indica que la tercera edición del COACH está en prensa, no habiendo noticias de Paul H. Brookes Publishing sobre una versión posterior al año 1998.

\section{DISCUSIÓN}

Como se señaló hacia el final del apartado titulado La conducta adaptativa en el campo de la discapacidad intelectual, se propone en lo que sigue un análisis que pretende puntualizar algunas observaciones críticas respecto de la integración del constructo de conducta adaptativa, a partir de la descripción de su uso en la política educativa. Con este propósito se desarrollará en primer lugar un examen del Decreto de Ley $\mathrm{N}^{\circ} 170$, luego se llevará a cabo un análisis de las Orientaciones Técnicas para, en tercer lugar, exponer observaciones relacionadas con los Formularios.

\subsection{SOBRE EL DECRETO LEY}

El Decreto de Ley $N^{o} 170$, en su artículo $N^{\circ} 63$, señala lo siguiente “...y cuando los resultados en las escalas anteriormente señaladas se encuentran a más de dos desviaciones estándar por debajo de la media...". La remisión a "escalas anteriormente señaladas" hace suponer que en el mismo Decreto, en algún apartado previo, están contenidas dichas "escalas". Este no es el caso. Inclusive si se considera que la expresión "escalas anteriormente señaladas" se refiere al Artículo inmediatamente anterior, el $\mathrm{N}^{\circ} 62$, en el que se alude a escalas para evaluar el funcionamiento intelectual; como se sabe éstas no contienen las mismas dimensiones que las escalas de conducta adaptativa.

Aún más, desde sus inicios, el propósito de la incorporación de la conducta adaptativa en el diagnóstico de la discapacidad intelectual consistió precisamente en equilibrar el énfasis excesivo de la medición en el funcionamiento intelectual, para generar así un diagnóstico lo menos discriminatorio posible. Lo antes señalado sugiere la existencia de un problema de comprensión, el cual se expresa en la equívoca redacción comentada, la que eventualmente puede conducir a errores en la práctica profesional.

De forma general, el punto que se quiere subrayar con esta observación, que pudiera parecer marginal, concierne a la relación entre investigación y política. Siguiendo la perspectiva de Bronfrenbrenner (1987) parece prudente revisar la forma convencional de tal relación en ciencias sociales, vale decir, aquella que "sostiene que la política social debería basarse en el conocimiento científico" (Bronfrenbrenner, 1987: 28). Pero no para proponer lo contrario sino con la finalidad de subrayar la necesidad de una relación sinérgica entre ambas que permita obtener el mejor provecho del vínculo entre una y otra para el desarrollo humano. 


\subsection{REFERENTE A LAS ORIENTACIONES TÉCNICAS}

En relación con las "Orientaciones Técnicas para la Evaluación Diagnóstica de Estudiantes que Presentan Necesidades Educativas Especiales Asociadas a Discapacidad Intelectual" podemos precisar que es un texto que se elabora y es publicado el año 2009 en la página oficial de internet del Ministerio de Educación de Chile. Si se visita el sitio ahora podrá verificarse que en cada una de las páginas del texto, en su parte superior, se agregó en rojo y con letra mayúscula la siguiente advertencia: "ESTE DOCUMENTO SE ENCUENTRA EN REVISIÓN POR LO QUE PODRÍA SUFRIR ALGUNAS MODIFICACIONES".

Cabe señalar que si bien la aparición del documento de Orientaciones Técnicas constituye un avance a nivel nacional en cuanto a la evaluación de la conducta adaptativa, éste aparece no obstante con una década de desfase en relación con el progreso internacional de la investigación teórica y empírica. Prueba de ello es, entre otros, el texto Adaptive Behavior and Its Measurement. Implications for the Field of Mental Retardation, del año de 1999, y el hecho de que la evaluación de la conducta adaptativa esté incorporada a la Ley sobre personas con discapacidad desde el año de 1975, en los Estados Unidos de Norteamérica.

El documento de Orientaciones Técnicas comienza diciendo que:

Se entenderá por discapacidad intelectual a la presencia de limitaciones sustantivas en el funcionamiento actual del estudiante, caracterizado por un desempeño intelectual significativamente por debajo de la media que se da en forma concurrente con limitaciones en su conducta adaptativa, manifestada en habilidades prácticas, sociales y conceptuales, y que comienza antes de los 18 años (Luckasson y cols., 2002: 8).

Si se confronta la referencia al texto de "Luckasson y cols., 2002: 8" podrá verificarse que en el texto original no se habla de discapacidad intelectual, sino más bien de retraso mental. El que éste sea el caso conlleva una confusión conceptual que consiste en que mientras que la expresión retraso mental es un lenguaje peyorativo, ambiguo y de etiquetado, la de discapacidad supone una comprensión de la situación de las personas desde un punto de vista socioecológico, que ejemplifica la interacción entre la persona y su entorno y que con los apoyos individualizados mejora el funcionamiento de la persona. A quienes trabajan en el campo académico y profesional, pero inclusive también para quienes no lo hacen, nos parece una dificultad derivada del conocimiento que justifica el uso de ambos constructos. Por otra parte, durante el año 2009 se da a conocer la décimo primera versión del Manual (Schalock et al., 2010) en el cual efectivamente se utiliza el concepto de discapacidad intelectual (p. 1), lo cual es coherente con la propuesta de cambio de constructo presentada por la AAIDD en enero del 2007.

De acuerdo con la información que se ha sintetizado antes respecto de "Instrumentos de evaluación de la conducta adaptativa sugeridos en Chile" (Tabla 1) y que forma parte del contenido de las Orientaciones Técnicas, conviene señalar que ninguna de las pruebas consignadas ofrece criterios operativos para establecer limitaciones significativas en el diagnóstico de discapacidad intelectual, esto es, la indicación de que el puntaje debe estar a dos desviaciones estándar bajo la media en una o en el puntaje global de las tres dimensiones que integran la conducta adaptativa, a saber, habilidades conceptuales, sociales y prácticas. No obstante, se trata de instrumentos útiles para evaluar la conducta adaptativa, lo que dada la situación nacional constituye un avance real. 
En lo referente a las tres escalas a las que se hace mención en el documento de Orientaciones Técnicas, vale decir, el ICAP, Vineland y SIS, ninguna de ellas ayuda a satisfacer el segundo criterio para el diagnóstico de la discapacidad intelectual. En el caso del Inventario para la Planificación de Servicios y Programación Individual (ICAP), que es un trabajo del Dr. Montero Centeno, junto con el Inventario de Destrezas Adaptativas (CALS) y el Currículum de Destrezas Adaptativas (ALSC), lo que conforma un sistema de valoración-enseñanza-evaluación de destrezas adaptativas de acceso frecuente tanto en España como en Iberoamérica; el ICAP, que con el índice de independencia general ofrece la posibilidad de entregar como resultado el grado de desviación estándar requerido en el contexto de la evaluación para el diagnóstico, no obstante carece de una densidad suficientemente alta como para ofrecer mayores garantías de certeza, dada la relevancia del diagnóstico de discapacidad intelectual y los sensibles efectos que tiene sobre la comprensión personal un diagnóstico eventualmente inexacto.

La segunda escala es la de Vineland, la cual se genera a partir de la tesis de pregrado para optar al título de psicólogo de Adriana Otero Quiroz, desarrollada en el año de 1959 en la Universidad de Chile. La Escala de Madurez Social de Vineland, que es el nombre completo del instrumento, al que se hace alusión en las Orientaciones Técnicas, fue construida originalmente en el año de 1935 por Edgar Doll, pionero en conducta adaptativa. La escala adaptada en Chile consideraba sólo aspectos lingüísticos, sin haber sido estandarizada, lo cual es manifestado por la propia autora en el apartado de sugerencias de la tesis, refiriéndose a ello de la siguiente manera: "Sería de gran interés estandarizar la Escala de Madurez de Vineland con el fin de transformarla en un instrumento de evaluación clínica y educacional en la práctica común" (Otero, 1959, p. 124). De hecho, la escala de Vineland de 1935, construida por Doll, fue ampliamente revisada y modificada en la versión de 1984 de la misma escala por Sparrow, Bala, \& Cicchetti.

Finalmente, la tercera escala, la Escala de Intensidad de Apoyos (SIS) tiene como finalidad:

a) identificar el perfil y la intensidad de las necesidades de apoyo de una persona, b) proporcionar una medida de las necesidades específicas de apoyo para la realización de planes individualizados de adaptación y apoyo, c) proporcionar una medida fiable para la supervisión de los progresos y los resultados de los planes de intervención o apoyo, y d) proporcionar información objetiva sobre las necesidades de apoyo para los estudios de costes, para la planificación y asignación de recursos en centros, organismos o comunidades y para el diseño de las políticas de protección social (Thompson et al., 2004: 21).

Los autores de la SIS (versión original, Thompson, Bryant, Campbell, Craig, Hughes, Rotholz, Schalock, Silverman, Tassé, \& Wehmeyer, 2004; traducción y adaptación española, Verdugo, Arias, \& Ibáñez, 2007) señalan que este instrumento y las escalas de conducta adaptativa miden constructos relacionados pero diferentes, y que han de ser utilizados con propósitos diferentes (Thompson et al., 2004). Indican además que son escalas que se complementan para brindar apoyos a las personas con discapacidad intelectual, pero por ningún motivo, como lo menciona el documento de Orientaciones Técnicas, es para evaluar la conducta adaptiva.

Otro punto es el que se refiere a los antecedentes bibliográficos que respaldan teóricamente al documento de Orientaciones Técnicas, los cuales son insuficientes en cantidad y no representan el nivel de la discusión científica que se había alcanzado 
hasta el año 2009. Por ejemplo, el documento no considera en el caso de Discapacidad Intelectual, las referencias siguientes: International Review of Research in Mental Retardation (2007); User's guide: Mental retardation. Definition, Classification and systems of supports - $10^{\text {th }}$ Edition (2007); What Is Mental Retardation? Ideas for an evolving Disability in the 21st Century (2006); Mental Retardation. Determining eligibility for social security benefits (2002); Manual of Diagnosis and Professional Practice in Mental Retardation (1996). En el mismo sentido, para el caso de la conducta adaptativa se omiten importantes contribuciones, tales como: ABAS-II. Clinical Use and Interpretation (2008a); Best Practices in the Assessment of Adaptive Behavior (2008b); La Conducta Adaptativa en el panorama científico y profesional actual (2005); Adaptive Behavior and Its Measurement. Implications for the Field of Mental Retardation (1999); Discapacidad y Conducta Adaptativa (1993); The Journal of Special Education. Especial Issue: adaptive Behavior (1987); Adaptive Behavior. Concepts \& Measurements (1978). Finalmente, sobre el Juicio clínico tanto para la evaluación del funcionamiento intelectual como para la conducta adaptativa, el texto Clinical Judgment (2005), el cual constituye una ineludible referencia para efectuar el juicio clínico en el campo de la discapacidad intelectual. El texto anterior, relativo a la ausencia de referencias bibliográficas, pretende contribuir a la elaboración consistente de un documento cuyo propósito consiste en servir de orientación para la adopción de decisiones profesionales adecuadamente fundamentadas, permitiendo con esto una mejor comprensión y aplicación de los constructos en cuestión.

En concreto, las Orientaciones Técnicas presentan cuatro (4) Referencias y todas ellas en español, cuyos años de publicación corresponden a 1994, 2001, 2002 y 2004. Tres de las referencias aludidas son de libre acceso a través de internet. Por contraste, en este mismo artículo se presentan once (11) referencias bibliográficas, siendo todas ellas posteriores a la última de las publicaciones referidas por las Orientaciones Técnicas. No deja de preocupar la falta de fundamentación y eventualmente la pertinencia de las referencias bibliográficas sobre las cuales se elaboran los documentos elaborados por el MINEDUC. Sobre el mismo punto, concerniente a las referencias bibliográficas, era necesario detenernos, puesto que las Orientaciones Técnicas elaboradas por la Unidad de Educación Especial del Ministerio de Educación de Chile y con la participación de profesionales de distintos ámbitos y grados académicos, habrían supuesto una base más representativa de la investigación actual al menos en lo que a la conducta adaptativa se refiere.

\subsection{SOBRE LOS FORMULARIOS ÚNICOS}

En relación a los "Formularios Únicos", en los casos de "diagnóstico de rango límite" y "discapacidad intelectual severa" se señala el "desempeño personal y social del estudiante" en diez áreas (las mismas de Luckasson et al., 1992, las que también son asumidas en el DSM IV TR, 1994/2000). Las categorías de evaluación que se usan son: Muy Bien, Bien, Regular y Deficiente. Sin embargo, las pruebas sugeridas no se corresponden con las categorías establecidas en el Formulario Único. Esto solo podría hacerse mediante una especulación por parte de quien evalúe la conducta adaptativa. 
En concordancia con los formularios únicos, las investigaciones indican que la evaluación de la conducta adaptativa es importante, además de ser uno de los tres criterios de diagnóstico de discapacidad intelectual, porque permite la planificación educativa y la generación de programas de apoyo para personas con necesidades educativas especiales de tipo transitorio o permanente (Matson, 2007; Ditterline \& Oakland, 2009).

En el contexto de las capacitaciones efectuadas por el MINEDUC en el año 2010, con el propósito de difundir el "Manual de Apoyo a Docentes: Educación de Estudiantes que Presentan Trastornos del Espectro Autista”, desafortunadamente se omitió la incorporación del Módulo 5, cuyo propósito es "Dar algunos apoyos desde la conducta adaptativa de la persona con trastorno autista, integrado en la escuela especial". De hecho, para remediar esta omisión, el módulo fue multicopiado y entregado a todos los participantes a sugerencia de la Mg. María del Carmen Aguilera, profesional que elaboró el Manual conjuntamente con personal del MINEDUC.

Acercándonos al final de este apartado, es justo destacar las iniciativas llevadas a cabo en Chile, en cuanto a instrumentos de evaluación. En este sentido se desarrolló un estudio sobre la adaptación y estandarización a nivel de la Provincia de Talca con el Inventario de Destrezas Adaptativas (CALS), que es un instrumento de evaluación de tipo criterial que permite una planificación de programas en torno a las habilidades de la vida diaria (Luckasson et al., 2002: 89).

Del mismo modo cabe mencionar que se está trabajando en Chile sobre la estandarización del Sistema de Evaluación de la Conducta Adaptativa (Adaptive Behavior Assessment System - ABAS II de Harrison \& Oakland, 2003). Este instrumento se caracteriza por sus altas propiedades psicométricas, el amplio rango de edad de 0 a 89 años y su precisión para evaluar grupos clínicos y no clínicos, diferenciando sus perfiles en fortalezas y debilidades presentadas por niños, adolescentes y adultos (Ditterline \& Oakland, 2009). Esta iniciativa se está llevando a cabo conjuntamente por la Universidad de Deusto, Bilbao - España, la editorial TEA Ediciones, Madrid - España y la Universidad Católica del Maule, Talca - Chile.

La iniciativa de avanzar en la incorporación de la conducta adaptativa como criterio de diagnóstico, por parte del Ministerio de Educación de Chile, específicamente de la Unidad de Educación Especial, sin duda que ha sido un acierto tanto para el diagnóstico, la clasificación y la generación de apoyos para las personas con discapacidad y discapacidad intelectual.

No obstante ello, a nivel nacional persisten varios desafíos: la estandarización de pruebas de conducta adaptativa, la determinación de cómo debe ser la evaluación de la conducta adaptativa conforme a las evidencias aportadas por la investigación, la integración de este constructo en la formación profesional brindada en el país por las instituciones de educación superior.

Estos y otros desafíos en coherencia con la evidencia aportada por la investigación y la práctica basada en la investigación, son los lineamientos que en un futuro cercano debe adoptar la política pública para la generación de servicios asociados a los derechos de las personas que presentan discapacidad y discapacidad intelectual. En este sentido, el constructo de conducta adaptativa y sus relaciones con el desarrollo personal requieren apoyos desde la atención temprana, la escolarización, la transición a la vida adulta y la plena adultez. Vale decir, la importancia del constructo radica en constituir una base para el desarrollo de políticas públicas que contribuyan a mejorar las condiciones de vida de las personas, sus funcionamientos y libertades efectivas. 


\section{AGRADECIMIENTOS}

Nuestro agradecimiento al Dr. Jorge Alarcón Leiva, por sus invalorables aportes a la discusión académica durante la elaboración del presente artículo.

\section{REFERENCIAS BIBLIOGRÁFICAS}

American Psychological Association (2010). Publication Manual of the American Psychological Association (6 $6^{\text {th }} E d$.). Washington, DC: American Psychological Association.

American Psychiatric Association (1994). Diagnostic and statistical manual for mental disorders $\left(4^{\text {th }} E d\right.$.). Washington, DC: Author.

American Psychiatric Association (2000). Diagnostic and statistical manual for mental disorders $T R\left(5^{\text {th }} E d\right.$.). Washington, DC: Author.

Baumeister, A. \& Muma, J. (1975). On defining mental retardation. The Journal of Special Education, 9 (3), 293-306.

Bronfenbrenner, U. (1987). La Ecología Del Desarrollo Humano. España: Paidos.

Bruininks, R., McGrew K. \& Marayuma, G. (1988). Structure of adaptive behavior in samples with and without mental retardation. American Journal of Mental Retardation, 3, 265-272.

Bruininks, R., Thurlow, M. \& Gilman, C. (1987). Adaptative behavior and mental retardation. The Journal of Special Education, 21, 1 69-88.

Clausen, J. (1972). The continuing problem of defining mental deficiency. The Journal of Special Education, 6, 97-106.

Coulter, A. \& Morrow, H. (1978). Adaptive Behavior: Concepts and Measurements. New York: Grune \& Stratton.

Ditterline, J. \& Oakland, T. (2009). Relationships Between Adaptive Behavior and Impairment. In S. Goldstein \& J. Naglieri (Eds.). Assessing Impairment. From Theory to Practice (pp. 3148). New York: Springer.

Eyman, R. \& Widaman, K. (1987). Lifespan development on institutionalized and community-based mentally retarded persons, revisited. American Journal of Mental Deficiency, 91, 559-569.

Ford, A., Schnorr, R., Meyer, L., Davern, L., Black, J. \& Dempsey, P. (1989). The Syracuse community referenced curriculum guide: for students with moderate and severe disabilities. Baltimore, MD: Brookes Publishing.

Giangreco, M., Cloninger, C. \& Iverson, V. (1998). Choosing Outcomes and Accommodations for Children. A guide to educational planning for Students with Disabilities $(\mathrm{COACH})\left(2^{\text {th }}\right.$ ed.). Maryland: Paul H. Brookes Publishing.

Gobierno de Chile, Ministerio de Educación. Decreto No 170. (2010).

Greenspan, S. (1981). Social Competence and Handicapped Individuals: Practical implications of a proposed model. Advances in Special Education, 3, 41-82.

Greenspan, S. \& Granfield, J. (1992). Reconsidering the construct of mental retardation: Implications of a model of social competence. American Journal of Mental Retardation, 96, 4, 442-453.

Greenspan, S., Switzky, H. \& Granfield, J. (1996). Everyday intelligence and adaptive behavior: A theoretical framework. In Jacobson, J.W. \& Mulick, J.A. (Eds.): Manual of diagnosis and professional practice in mental retardation (pp. 127-135). Washington: American Psychological Association.

Greenspan, S. \& Driscoll, J. (1997). The role of intelligence in a broad model of personal competence. In Flanagan, D.P., Genshaft, J.L. y Harrison, P. Contemporary intellectual Assessment: theories, test and issues. (pp. 131-150). New York: The Guilford Press.

Grossman, H. (1983). Classification in mental retardation. Washington, DC: American Association on Mental Deficiency. 
Harrison, P. \& Oakland, T. (2003). Adaptive Behavior Assessment System (2 ${ }^{\text {th }}$ Ed.). Los Angeles, CA: Western Psychological Services.

Harrison, P. \& Raineri, G. (2008b). Best practices in the assessment of adaptive behaviour. In A. Thomas \& J. Grimes (Eds.) Best practices in school psychology (5 ${ }^{\text {th }}$ Ed.) (pp. 605-616) Bethesda, MD: NASP Press.

Hawkins, G. \& Cooper, D. (1990). Adaptive behavior measures in mental retardation research: Subject description in AJMD/AJMR articles. American Journal of Mental Retardation, 94, 654-660.

Heber, R. (1959). A Manual on terminology and classification in mental retardation. American Journal of Mental Deficiency, Monograph Suplement, 64.

Horn, E. \& Fuchs, D. (1987). Using adaptive behavior in assessment and intervention: An overview. Journal of Special Education, 21, 11-26.

ICE. (1993). Discapacidad y Conducta Adaptativa. Bilbao: Universidad de Deusto.

Janicki, M. \& Jacobson, J. (1986). Generation trends in sensory, physical and behavioral abilities among older mentally retarded persons. American Journal of Mental Deficiency, 90, 490-500.

Jacobson, J. \& Mulick, J. (2003). Defining Mental Retardation or the Incurability of Ideological Imbecility. In Switzky, H. \& Greenspan, S. (Eds.), What is Mental Retardation? Ideas for an Evolving Disability (pp. 230-241). Washington, DC: AAMR.

Jacobson, J. (2006). Focusing Comprehensive Functional Assessment: A Case Formulation of Dual Diagnosis Treatment Priorities Using ICF (ICIDH-2). In Switzky, H. \& Greenspan, S. (Eds.), What is Mental Retardation? Ideas for an Evolving Disability in the $21^{\text {st }}$ Century. Revised and Updated Edition (pp. 325-340). Washington, DC: AAMR.

Luckasson, R., Coulter, D., Polloway, E., Reiss, S., Schalock, R., Snell, M., Spitalnik, D. \& Stark, J. (1992). Mental retardation. Definition, Classification and Systems of Supports ( $9^{\text {th }}$ Ed.). Washington: American Association on Mental Retardation.

Luckasson, R., Borthwick-Duffy, S, Buntinx, W., Coulter, D., Craig, E, Reeve, A., Schalock, R., Snell, M., Spitalnik, D., Spreat, S. \& Tassé, M. (2002). Mental Retardation: Definition, Classification, and Systems of Supports. (10 ${ }^{\text {th }}$ Ed.). Washington D.C.: AAMR.

Matson, J. (2007). International Review of Research in Mental Retardation. Handbook of Assessment in Persons with Intellectual Disability, $\mathrm{N}^{\circ} 34$.

Mercer, J. (1973). Labeling the mentally retarded: Clinical and social system perspectives on mental retardation. Berkeley: University of California Press.

Meyers, C., Nihira, K., \& Zetlin, A. (1979). The measurement of adaptive behavior. In Ellis, N.R. (Ed.), Handbook of mental deficiency: Psychological theory and research $\left(2^{\text {th }}\right.$ Ed.) (pp. 431481). Hillsdale, NJ: Lawrence Erlbaum.

Montero, D. (1996). Evaluación de la Conducta Adaptativa en Personas con Discapacidad. Adaptación y validación del ICAP. Bilbao: Mensajero.

Montero, D. (2003). Conceptos clave: conducta adaptativa y autodeterminación. En Gobierno de Navarra. Dep. de Educación y Cultura. I Congreso Nacional de Educación y Personas con Discapacidad: "Conciencia, Compromiso y Mejora Continua". Navarra: Gobierno de Navarra.

Montero, D. (2005). La Conducta Adaptativa en el panorama científico y profesional actual. Intervención Psicosocial, 14 (3), 277-293.

Morreau, L., Bruininks, R. \& Montero, D. (2002). Inventario de Destrezas Adaptativas (CALS). Bilbao: Mensajero.

Nihira, K., Foster, R., Shellhaas, M. \& Leland, H. (1968). Adaptive behavior checklist. Washington, DC: American Association on Mental Deficiency.

Nihira, K. (1999). Adaptive behavior: A Historical Overview. In Schalock, R. (Ed.) Adaptative behavior and measurement. Implications for the field of de mental retardation (pp. 7-14). Washington: American Association on Mental Retardation. 
Organización Mundial de la Salud (2001). Clasificación Internacional del Funcionamiento, de la Discapacidad y de la Salud (CIF). Madrid: Ministerio de Trabajo y Asuntos Sociales. Secretaría General de Asuntos Sociales. Instituto de Migraciones y Servicios Sociales.

Orientaciones Técnicas para la Evaluación Diagnóstica de Estudiantes que presentan Necesidades Educativas Especiales Asociadas a Discapacidad Intelectual (documento de trabajo) Ministerio de Educación de Chile (2009).

Otero, A. (1959). La Escala de Madurez Social de Vineland. Primeros ensayos de aplicación. Tesis para optar al Título de Psicólogo, Universidad de Chile, Santiago, Chile.

Oakland, T. \& Harrison, P. (2008a). ABAS-II. Clinical Use and Interpretation. New York: Academic Press.

Schalock, R. (1999). Adaptive Behavior and Its Measurement: Setting the Future Agenda. In R. Schalock (Ed.), Adaptive Behavior and Its Measurement. Implications for the Field of Mental Retardation (pp. 209-222). Washington, DC: AAMR.

Schalock, R. (1999). The Merging of Adaptive Behavior and Intelligence: Implications for the Field of Mental Retardation. In R. Schalock (Ed.), Adaptive Behavior and Its Measurement. Implications for the Field of Mental Retardation (pp. 43-59). Washington, DC: AAMR.

Schalock, R. (2001). Conducta adaptativa, competencia personal y calidad de vida. En M.A. Verdugo y Jordán de Urríes (Coords.) Apoyos, Autodeterminación y Calidad de Vida. Actas de las IV Jornadas Científicas de Investigación sobre personas con discapacidad. Salamanca: Amarú.

Schalock, R. (2003). Mental retardation: A condition characterized by significant limitations in practical, conceptual, and social skills. In Switzky, H. \& Greenspan, S. (Eds.), What is mental retardation? Ideas for an evolving disability (pp. 363-378). Washington, DC: AAMR.

Schalock, R. \& Luckasson, R. (2005) Clinical Judgment. Washington, DC: AAMR.

Schalock, R., Buntinx, W., Borthwick-Duffy, S., Luckasson, R., Snell, M., Tassé, M. \& Wehmeyer, M. (2007). User's guide: Mental Retardation. Definition, classification and systems of supports (10 ${ }^{\text {th }} E d$.). Washington, DC: AAIDD.

Schalock, R., Borthwick-Duffy, S., Bradley, V., Buntinx, W., Coulter, D., Craig, E., Gomez, S., Lachapelle, Y., Luckasson, R., Reeve, A., Shogren, K., Snell, M., Spreat, S., Tassé, M., Thompson, J., Verdugo-Alonso, M., Wehmeyer, M. \& Yeager, M. (2010). Intellectual Disability Definition, Classification and Systems of Supports (1 1 th $^{\text {E }}$ ). Washington: AAIDD.

Silverman, W., Silver, E., Sersen, E., Lubin, R. \& Schwart, A. (1986). Factors related to adaptive behavior changes among a mentally retarded, physically disabled person. American Journal of Deficiency, 90, 651-658.

Sparrow, S., Bala, D. \& Cicchetti, D. (1984). Vineland Adaptive Behavior Scales, Circle Pines, MN: American Guidance Service.

Stancliffe, R. \& Abery, B. (2003). An ecological theory of self-determination: research evidence. In Wehmeyer, M., Abery, B., Mithaug, D. \& Stancliffe, R. (Eds.) (2003). Theory in selfdetermination. Foundations for Educational Practice (pp. 79-118). Illinois: Charles C. Thomas Publisher, Ltd.

Thompson, J., McGrew, K. \& Buininks, R. (1999). Adaptive and Maladaptive Behavior: Functional and Structural Characteristics. In R. Schalock (Ed.) (1999). Adaptive Behavior and its Measurement. Implications for the Field of Mental Retardation (pp. 15-42). Washington: American Association on Mental Retardation.

Thompson, J., Bryant, B., Campbell, E., Craig, E., Hughes, C., Rotholz, D., Schalock, R., Silverman, W., Tassé, M. \& Wehmeyer, M. (2004). Supports Intensity Scale. Washington DC: AAIDD.

Unidad de Educación Especial, Ministerio de Educación. (2010). Manual de Apoyo a Docentes: Educación de Estudiantes que presentan Trastornos del Espectro Autista. Santiago de Chile: MINEDUC.

Verdugo, M. \& Schalock, R. (2001). El concepto de calidad de vida en los servicios humanos. En Verdugo, M.A. y Jordán de Urríes, B. (Coords.) Apoyos, Autodeterminación y Calidad de 
Vida. Actas de las IV Jornadas Científicas de Investigación sobre Personas con Discapacidad. Salamanca: Amarú.

Verdugo, M., Arias, B. \& Ibáñez, A. (2007). Escala de Intensidad de Apoyos. SIS. Madrid: TEA Ediciones.

Wehmeyer, M., Abery, B., Mithaug, D. \& Stancliffe, R. (2003). Theory in self-determination. Foundations for educational practice. Illinois: Charles C. Thomas Publisher, Ltd.

Widaman, K., Gibbs, K. \& Geary, D. (1987). Structure of adaptive behavior: Replication across fourteen samples of nonprofoundly mentally retarded people. American Journal of Mental Deficiency, 91, 348-360.

Widaman, F. \& McGrew, S. (1996). The structure of adaptive behavior. In Jacobson, J.W. \& Mulick, J.A. (Eds.), Manual of diagnosis and professional practice in mental retardation (pp. 97-110). Washington, DC: American Psychological Association.

Witt, J. \& Martens, B. (1984). Adaptive Behavior: Test and assessment issues. Scholl Psyghology Review, 13, 215-230.

Wolfensberger, W. (1972). The principle of Normalization in Human Services. Toronto: National Institute on Mental Retardation.

Woodcock, R. \& Muñoz-Sandoval, A. (1993). Conducta Adaptativa y Teoría de la Inteligencia. En ICE, Discapacidad y Conducta Adaptativa (pp. 41-49). Bilbao: Universidad de Deusto.

Zigler, E., Balla, D. \& Hodapp, R. (1984). On the definition and classification of mental retardation. American Journal of Mental Deficiency, 89, 3, 215-230. 
